\title{
$\left[{ }^{18}\right.$ F]Fluorodeoxyglucose-positron emission tomography screening for lung cancer: a systematic review and meta-analysis
}

\author{
Chun-Ru Chien a,e,1, Ji-An Liang ${ }^{\mathrm{a}, \mathrm{e}, 1}$, Jin-Hua Chen ${ }^{\mathrm{f}, 1}$, Hsiao-Nin Wang ${ }^{\mathrm{c}}$, Cheng-Chieh Lin ${ }^{\mathrm{b}, \mathrm{e}}$, \\ Chih-Yi Chen ${ }^{c}$, Pin-Hui Wang ${ }^{c}$, Chia-Hung Kao ${ }^{d, e}$, Jun-Jun Yeh ${ }^{g}$
}

${ }^{a}$ Department of Radiation Oncology, China Medical University Hospital, Taichung, Taiwan; ${ }^{b}$ Department of Community Medicine and Health Examination Center, China Medical University Hospital, Taichung, Taiwan; ${ }^{c}$ Cancer Center, China Medical University Hospital, Taichung, Taiwan; ${ }^{d}$ Department of Nuclear Medicine and PET Center, China Medical University Hospital, Taichung, Taiwan; ${ }^{e}$ School of Medicine, College of Medicine, China Medical University, Taichung, Taiwan; ${ }^{f}$ Biostatistics Center and School of Public Health, Taipei Medical University, Taipei, Taiwan; ${ }^{g}$ Departments of Family Medicine and Chest Medicine, Ditmanson Medical Foundation Chia-Yi Christian Hospital, Chiayi, Taiwan; Chia Nan University of Pharmacy and Science, Tainan, Taiwan; Meiho University, Pingtung, Taiwan

Corresponding address: Chia-Hung Kao, Department of Nuclear Medicine and PET Center, China Medical University Hospital, No. 2, Yuh-Der Road, Taichung 404, Taiwan.

Email: d10040@mail.cmuh.org.tw.

Jun-Jun Yeh, MD, Departments of Family Medicine and Chest Medicine, Ditmanson Medical Foundation Chia-Yi Christian Hospital, No. 539, Zhongxiao Road, Chiayi City 600, Taiwan.

Email: anvin. funlan@msa.hinet.net.

${ }^{I}$ C.R. Chien, J.A. Liang and J.H. Chen contributed equally to this work.

Part of this work has been presented at the ISPOR 16th Annual International Meeting, Baltimore, MD, May 21-25, 2011; and The European Multidisciplinary Cancer Congress 2011, Stockholm, September 23-27, 2011.

Date accepted for publication 29 August 2013

\begin{abstract}
Rationale and objectives: Although low-dose computed tomography (CT) is a recommended modality for lung cancer screening in high-risk populations, the role of other modalities, such as $\left[{ }^{18} \mathrm{~F}\right]$ fluorodeoxyglucose-positron emission tomography (PET), is unclear. We conducted a systematic review to describe the role of PET in lung cancer screening. Materials and methods: A systematic review was conducted by reviewing primary studies focusing on PET screening for lung cancer until July 2012. Two independent reviewers identified studies that were compatible for inclusion/ exclusion criteria. The analysis was restricted to English and included studies published since 2000. A descriptive analysis was used to summarize the results, and the pooled diagnostic performance of selective PET screening was calculated by weighted average using individual sample sizes. Results: Among the identified studies $(n=3497), 12$ studies were included for analysis. None of the studies evaluated the efficacy of primary PET screening specific to lung cancer. Eight studies focused on primary PET screening for all types of cancer; the detection rates of lung cancer were low. Four studies reported evidence of lung cancer screening programs with selective PET, in which the estimated pooled sensitivity and specificity was $83 \%$ and $91 \%$, respectively. Conclusions: The role of primary PET screening for lung cancer remains unknown. However, PET has high sensitivity and specificity as a selective screening modality. Further studies must be conducted to evaluate the use of PET or PET/computed tomography screening for high-risk populations, preferably using randomized trials or prospective registration. Advances in knowledge: Our meta-analysis indicates that PET has high sensitivity and specificity as a selective screening modality.
\end{abstract}

Keywords: Lung cancer; positron emission tomography; cancer screening. 


\section{Introduction}

Lung cancer is one of the major cancer-related causes of death worldwide. Low-dose computed tomography (CT) has been the recommended screening modality for highrisk populations since $2011^{[1,2]}$.

$\left[{ }^{18} \mathrm{~F}\right]$ Fluorodeoxyglucose (FDG)-positron emission tomography (PET) combined with CT is helpful in the staging, imaging, and prognosis of patients with lung cancer ${ }^{[3-6]}$. It is crucial to differentiate equivocal primary or regional lesions and to detect distant metastasis. PET has been used to effectively detect occult metastases, which may manifest in the initial stage, in a large number of patients with lung cancer. The evaluation of prognosis, including that for small tumors $(<3 \mathrm{~cm})$, can be conducted ${ }^{[7]}$. It is also a useful tool for early detection of lung cancer and pulmonary nodules not specifically related to screening ${ }^{[8,9]}$. A recent meta-analysis indicated that the sensitivity and specificity in this scenario is $95 \%$ and $82 \%$, respectively ${ }^{[9]}$. Considering the potential of PET as an effective screening measure, it can be used as a primary procedure or in combination with CT. However, the role of PET in primary screening ${ }^{[10]}$ and in evolution of work-up for CT-detected nodules (i.e., selective screening) remains unclear ${ }^{[1]}$; therefore, a systematic review was performed to evaluate the role of PET in lung cancer screening.

\section{Materials and methods}

\section{Literature search and selection criteria}

We searched for primary studies focusing on PET screening for lung cancer using the following keywords: lung cancer AND positron emission tomography AND screen OR screening in PubMed until July 2012, similarly to a previous CT lung cancer screening systematic review ${ }^{[11]}$. All studies were independently reviewed by 2 reviewers (Reviewer 1: C.R. Chien; Reviewer 2: H.N. Wang or P.H. Wang) to identify studies that were compatible with the 3 inclusion/exclusion criteria: (1) primary research; (2) papers that focused on screening for lung cancer; and (3) PET with and without comparison as part(s) of the screening modality. A third reviewer (C.H. Kao) was considered for consensus in the event of a disagreement between the 2 reviewers. Manual searching for relevant cases was also performed for the included studies. The analysis was restricted to English and non-overlapping studies published since 2000. If overlapping patient cohorts were used between multiple studies, the latest or largest study was included.

\section{Data extraction and quality assessment}

Data were obtained for author, year of publication, study design, study period, study country, risk factor, study population characteristics, identification of lung cancer cases, and contributions from PET for all included studies. Data were extracted independently by 2 investigators (C.R. Chien and C.H. Kao), and discrepancies were resolved by consensus. After an initial evaluation, papers in final analysis were divided into 2 categories: (1) primary PET: studies focused on primary PET screening for cancer; and (2) selective PET: studies that reported findings in lung cancer CT screening programs with selective PET. The primary PET studies mostly lacked information regarding false-positive or false-negative results, except for 2 studies providing information on PET screening ${ }^{[12]}$ and $\mathrm{PET} / \mathrm{CT}^{[13]}$, respectively. Subsequently, a methodological quality analysis and meta-analysis were performed for selective PET studies because of the limited available data. Methodological quality was assessed using the updated QUADAS-2 tools ${ }^{[14]}$.

\section{Statistical analysis}

The results from each study were tabulated and summarized using descriptive analysis. Data on sensitivity, specificity, and accuracy of selective PET screening for lung cancer were calculated from the original numbers provided in the 4 selective PET studies. The data sets were pooled from the true-positive, false-positive, true-negative, and false-negative results from the relevant studies. The pooled sensitivity and specificity were calculated by weighted average of these statistics. The weights are the sample size of each study ${ }^{[15]}$. When the estimation of sensitivities and specificities for each study was at least one zero cell, a correction of $1 / 2$ was added to every cell for the study to define the estimators. We also tested the threshold effect using the Spearman correlation coefficient between sensitivity and specificity. However, the result was non-significant and was not displayed in the pooled analysis. We attempted to fit each set of data to a summarized receiver-operating characteristic (sROC) curve, and calculate the area under the sROC curve (AUC). To examine the publication bias, we used the Deeks funnel plot using the linear regression method, which describes the association between diagnostic log odds ratio against sample size. Statistical analyses were conducted for these calculations using Meta-Disc version 1.4, a free statistical software package (Unit of Clinical Biostatistics, Ramón y Cajal Hospital, Madrid, Spain), and Stata version 11 (StataCorp, College Station, TX, USA) ${ }^{[16]}$.

\section{Results}

\section{Literature search}

The flowchart of the literature search is shown in Fig. 1. A total of 3497 searches were conducted, including database searching and manual searching. Among the identified cases, 238 studies published before 2000 were excluded because of technology advancements in the 


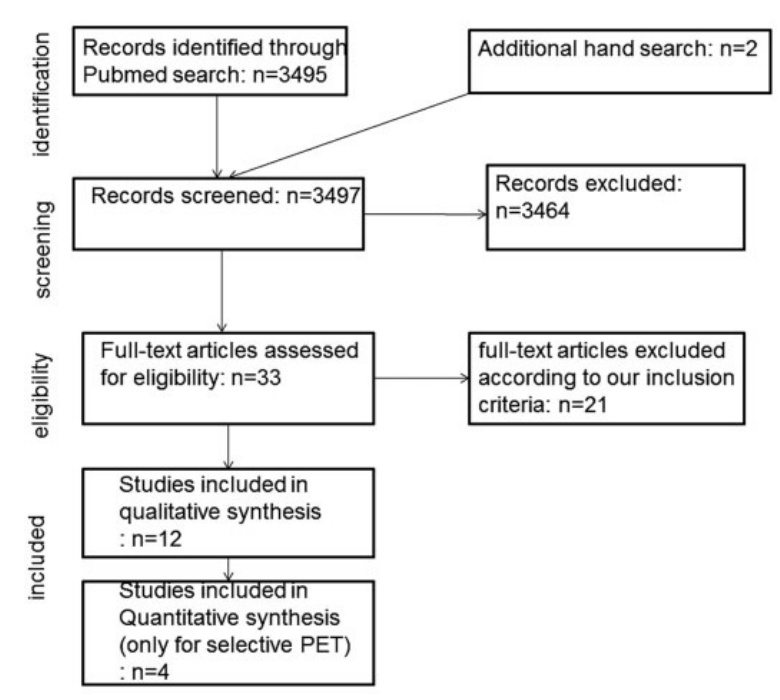

Figure 1 Flowchart of literature search.

past 10 years, 3111 cases were excluded because of irrelevant titles and keywords, and 115 papers were excluded after reviewing the abstracts. A complete evaluation of papers led to the exclusion of 21 studies, with 12 studies remaining for analysis ${ }^{[12,13,17-26]}$. One selective PET study was not included in the final analysis because of limited use of PET and the statistically significant imbalance regarding tumor size among PET users and nonusers $^{[27]}$.

\section{Summary of studies included in general}

The studies included did not evaluate the efficacy of primary PET screening specifically for lung cancer. Eight studies focused on primary PET screening for cancer (Table 1$)^{[12,13,17-22]}$, and 4 studies reported findings from lung cancer CT screening programs with selective PET (Table 2) ${ }^{[23-26]}$.

\section{Summary of primary PET screening stu- dies: detection rates of all types of cancer, including lung cancer}

All primary PET screening studies (Table 1) were singlearm (i.e., no comparator) studies performed in Far East Asian countries (Japan, Taiwan, and Korea). Only 3 studies were conducted prospectively ${ }^{[12,21,22]}$. Only 2 studies were not single-round studies (i.e., PET was performed once as prevalent screening $)^{[17,21]}$. In addition to primary PET, all cases used other examination procedures, such as CT, as the screening modalities. The percentage of male participants among individual studies ranged from $51 \%$ to $70 \%$. The mean age of participants in individual studies ranged from 47 to 60 years. Lung cancer risk factors, such as history of smoking, were only reported in 2 studies $^{[20,21]}$. The detection rates for all types of cancer, including lung cancer, for each study are shown in Table 1. Among all primary PET studies (Table 1), 640 cancer cases were identified in the prevalent screen by a screening program (detection rate of $2 \%$ ), and 363 cases (detection rate of $1.13 \%$ ) were identified using PET. For lung cancer, 105 cases were identified in the prevalent screen by a screening program (detection rate of $0.33 \%$ ), and 58 cases (detection rate $0.18 \%$ ) were identified using PET.

\section{Summary of selective PET screening stu- dies: diagnostic performance}

Among the 4 selective PET studies (Table 2), most were prospective single-arm studies performed for a high-risk population (heavy smokers) in South Europe. The primary screening modality in these studies was chest CT, whereas PET was reserved for specific CT findings, such as large $(7-10 \mathrm{~mm})$ or growing lesions in the follow-up examination. The percentage of male participants among individual studies ranged from $56 \%$ to $74 \%$. The mean age of participants in individual studies ranged from 55 to 58 years. A quality assessment of diagnostic performance showed acceptable quality in these studies (Fig. 2). PET evaluation was performed on approximately $3 \%$ of participants in these trials. The diagnostic performance of selective PET in these studies is shown in Table 2. The estimated pooled sensitivity and specificity (with 95\% confidence interval) was 83\% (approximately $75 \%-89 \%$ ) and 91\% (approximately 86\%-95\%), respectively (Fig. 3). The heterogeneity chi-squared tests were not statistically significant. The $P$ values were 0.14 and 0.52 for pooled sensitivity and specificity, respectively (Fig. 3). The AUC value (0.945) was close to 1, which indicates that selective PET for lung cancer screening has a high diagnostic performance (Fig. 4).

\section{Discussion}

Low-dose CT is the current recommended modality for lung cancer screening for high-risk populations in the current National Comprehensive Cancer Network guideline. However, the role of PET in related work-up remains unclear. PET is currently considered for solid or semisolid nodules of at least $8 \mathrm{~mm}$ in size in prevalent screening, or enlarged nodules in incident screening ${ }^{[1]}$. Our systemic review provides an up-to-date summary of the relevant evidence regarding the role of PET for lung cancer screening. The role of primary PET screening for lung cancer remains unknown. PET may be used as a screening modality; however, it may not be suitable for lung cancer because the detection rate of lung cancer is low. PET can also be used as a selective modality in combination with CT for lung cancer screening in highrisk populations because it has high diagnostic performance; however, the prevalence of lung cancer in highrisk populations (prescreened by CT) is high. This study 


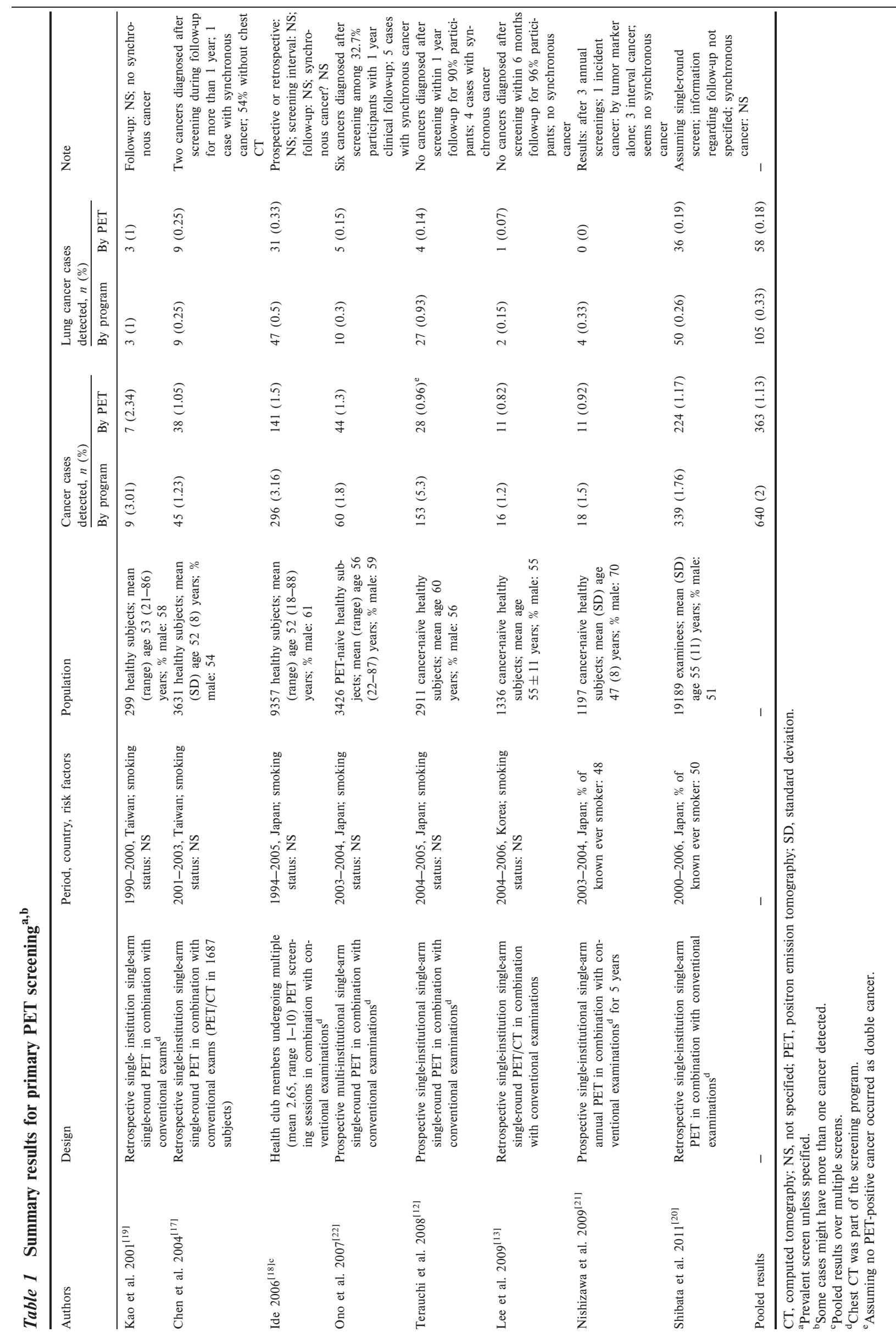


is the first to conduct a systematic review to examine the role of PET specifically for lung cancer screening.

The results indicate that PET is helpful in lung cancer (early) diagnosis, staging, evaluation of treatment response, and evaluation for single pulmonary nodules in patients who are not always asymptomatic ${ }^{[3-6,8,9]}$; however, we extended its role to screen detected nodules. The implication of our findings may also be significant. Lung cancer screening with low-dose CT is a complex and controversial issue ${ }^{[1,28,29]}$, and may not be cost-effective if used as a stand-alone modality ${ }^{[30]}$. A crucial concern is the unnecessary operations performed because of screening. In one modeling study ${ }^{[31]}$, CT screening led to 10 lung resections without any significant difference in lung cancer mortality. In another phase 3 trial $^{[32]}, 77$ patients in the CT arm received surgery, compared with 28 patients in the control group. Health care utilization often increases because of potential false-positive or indeterminate screening results ${ }^{[33]}$. The high diagnostic performance (AUC value 0.945) of selective PET screening observed in this study may be helpful regarding this issue. In addition, the detection rate of lung cancer using primary PET was low $(0.18 \%)$; this may have occurred because the lung tumors were small and may not be easily detectable using primary (unselected) PET. The low detection rate may also be partly attributed to the use of the FDG-PET scan in the majority of the included studies instead of the current hybrid PET/CT. The diagnostic performance of PET/CT in this setting (primary screening) must be examined in future studies.

This study had several limitations, the first of which was the external validity because of the strong geographic distribution tendency in the identified studies. All identified primary PET screening studies were conducted in Far East Asian countries (Japan, Taiwan, and Korea), whereas all the identified selective PET studies were conducted in Europe (Italy, Spain, and Denmark). Because of the substantial ethnic differences observed in patients with lung cancer in recent years ${ }^{[34]}$, as well as the potential effect of regional variation of granulomatous disease (a major cause of false-positive PET) ${ }^{[35]}$, it is unclear whether the results of this study can be applied to other regions; this must be validated in future studies. The second limitation is that most of the selective PET screening studies used PET instead of PET/CT ${ }^{[23,24,26]}$. However, this implied that the diagnostic performance of the current common practice (PET/CT) may be superior to our estimates. Therefore, our results may be not applicable to PET/CT, and the role of PET/CT screening must be further evaluated in future studies. The third limitation is that risk factors, such as smoking, were not adequately reported in most studies. The fourth limitation is that some heterogeneity may have occurred among the studies included in our meta-analysis, such as differences in patient populations, PET indications, diagnostic criteria, and PET technology; however, the heterogeneity tests were not statistically significant. The fifth limitation is 


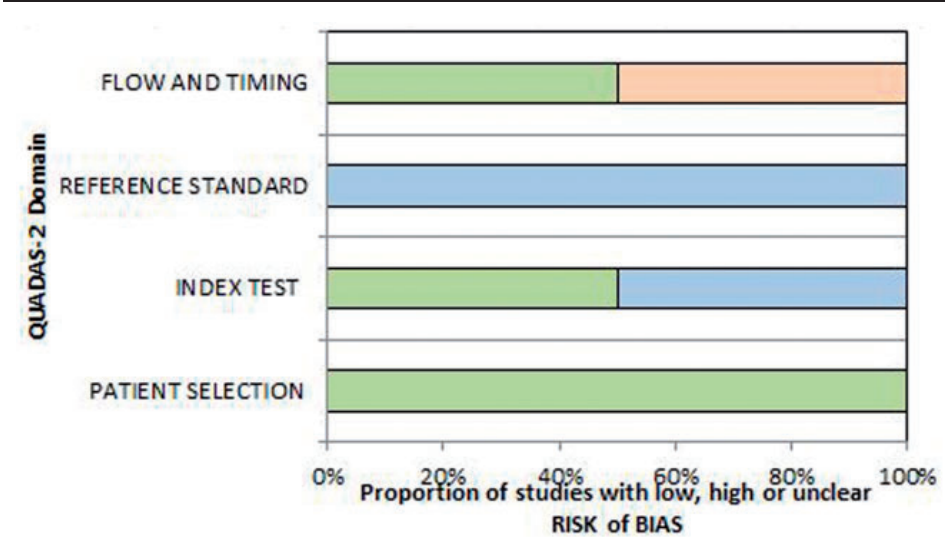

¿Low $\square$ High $\square$ Unclear

Figure 2 Quality assessments for selective PET studies.

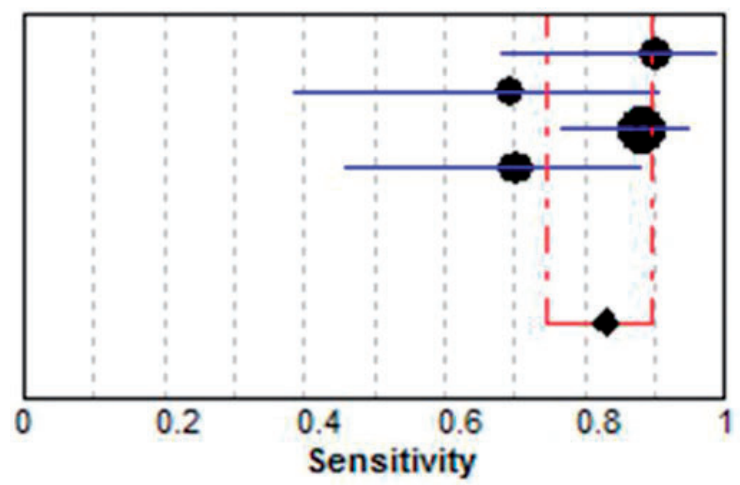

Pastorino $U$ et al 2003

Bastarrika G et al 2005

Sensitivity $(95 \% \mathrm{Cl})$

Veronesi $\mathrm{G}$ et al 2008

$0.90 \quad(0.68-0.99)$

$0.69 \quad(0.39-0.91)$

Ashraf $\mathrm{H}$ et al 2011

$0.88 \quad(0.77-0.95)$

$0.70 \quad(0.46-0.88)$

Pooled Sensitivity $=0.83(0.75$ to 0.89$)$

Chi-square $=5.41 ; \mathrm{df}=3(\mathrm{p}=0.1442)$

Inconsistency (l-square) $=44.5 \%$

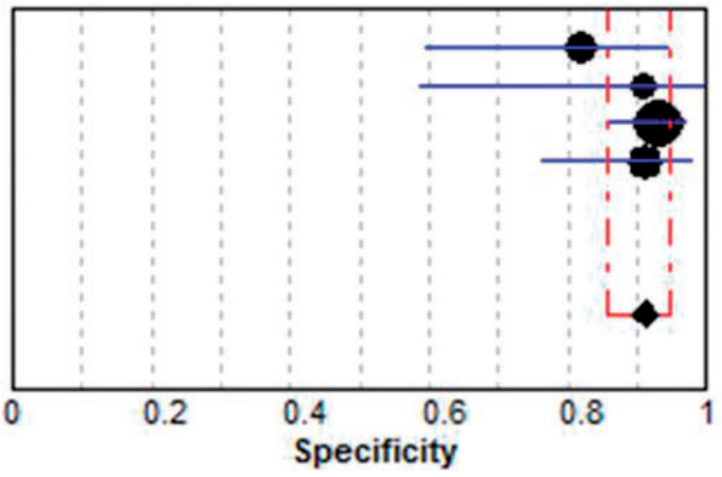

Pastorino U et al 2003

Bastarrika G et al 2005

Veronesi $\mathrm{G}$ et al 2008

Ashraf $\mathrm{H}$ et al 2011

Specificity $(95 \% \mathrm{Cl})$

$0.82(0.60-0.95)$

$0.91 \quad(0.59-1.00)$

$0.93 \quad(0.86-0.97)$

$0.91 \quad(0.76-0.98)$

Figure 3 Pooled sensitivity and specificity for selective PET screening. CI, confidence interval.

the potential publication bias because the $P$ value in the Deeks funnel plot was 0.09 . Therefore, the summary sensitivity and specificity must be interpreted with caution.

Despite these limitations, this study offers a unique contribution to the current trend, and showed that guidelines are required for the clinical work-up of indeterminate nodules identified using CT screening programs ${ }^{[28]}$; this study provides a strong rationale for the consideration of PET in these work-ups.

\section{Conclusion}

The role of primary PET screening for lung cancer remains unknown. However, PET has high sensitivity and specificity as a selective screening modality (i.e., for diagnosing lung cancer in patients with a pulmonary nodule found using CT screening). Further studies to evaluate the use of PET or PET/CT screening for highrisk populations must be conducted, preferably using randomized trials or prospective registration. 


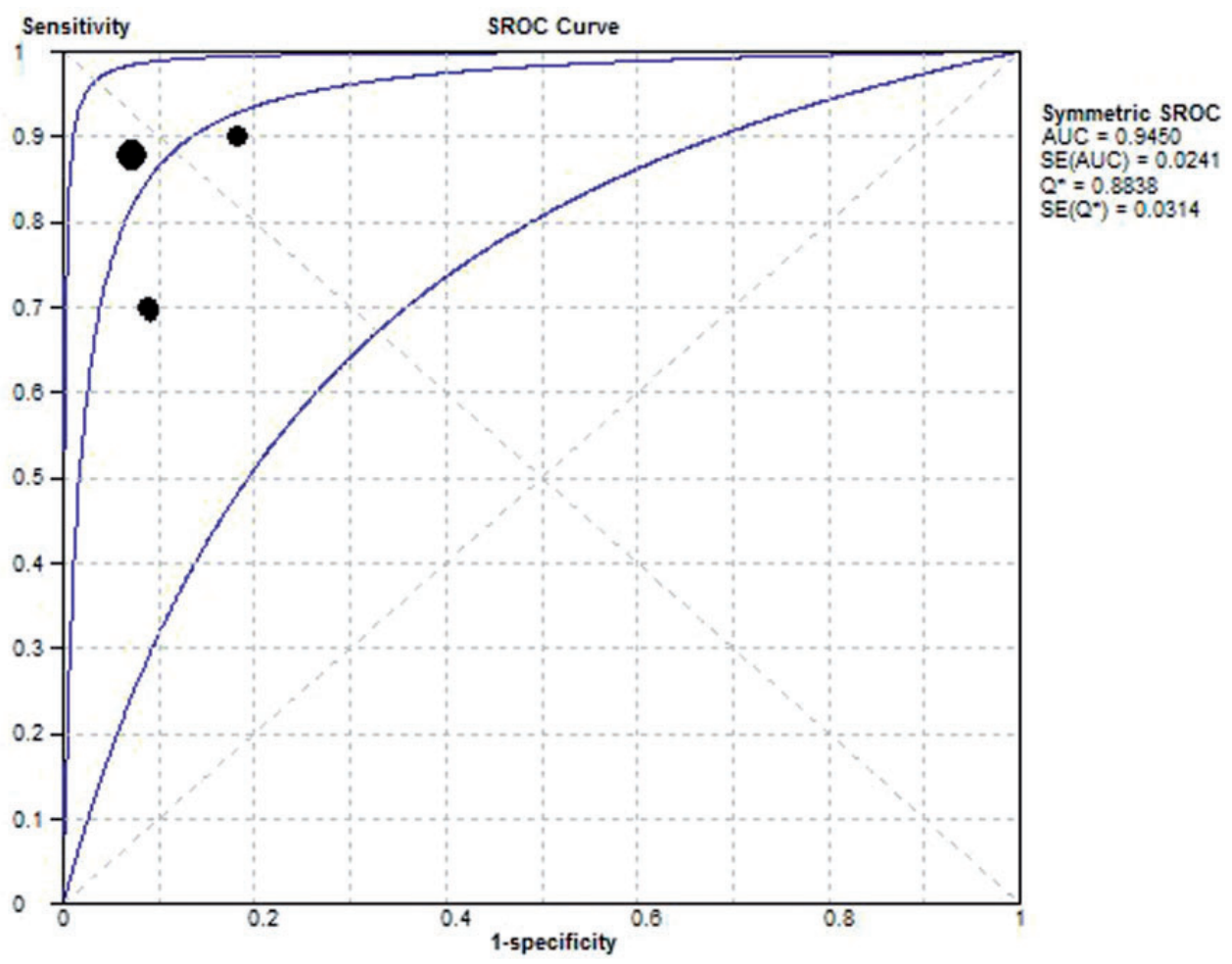

Figure 4 Summary receiver-operating characteristic (sROC) curves and 95\% confidence intervals of selective PET screening.

\section{Acknowledgements}

We would like to thank the Taiwan National Science Council (NSC 98-2314-B-039-014-MY3), the Taiwan Department of Health Clinical Trial and Research Center for Excellence (DOH102-TD-B-111-004), and the Taiwan Department of Health Cancer Research Center for Excellence (DOH102-TD-C-111-005).

\section{Conflict of interest}

The authors have no conflicts of interest to declare.

\section{References}

[1] NCCN clinical practice guidelines in oncology: Lung Cancer Screening 2013.v.1. Available from : http://www.nccn.org/professionals/physician_gls/pdf/lung_screening.pdf (accessed 11 July 2012).

[2] Aberle DR, Adams AM, Berg CD, et al. Reduced lung-cancer mortality with low-dose computed tomographic screening. $\mathrm{N}$ Engl J Med 2011; 365: 395-409. doi: 10.1056/ NEJMoa1102873. PMid: 21714641.

[3] MacManus M, Hicks RJ. The use of positron emission tomography (PET) in the staging/evaluation, treatment, and follow-up of patients with lung cancer: a critical review. Int J Radiat Oncol Biol Phys 2008; 72: 1298-1306. doi: 10.1016/ j.ijrobp.2008.08.022. PMid: 19028270.

[4] Paesmans M, Berghmans T, Dusart M, et al. Primary tumor standardized uptake value measured on fluorodeoxyglucose positron emission tomography is of prognostic value for survival in non-small cell lung cancer: update of a systematic review and meta-analysis by the European Lung Cancer Working Party for the International Association for the Study of Lung Cancer Staging Project. J Thorac Oncol 2010; 5: 612-619. doi: 10.1097/ JTO. 0b013e3181d0a4f5. PMid: 20234323.

[5] Kligerman S, Digumarthy S. Staging of non-small cell lung cancer using integrated PET/CT. AJR Am J Roentgenol 2009; 193: 1203-1211. doi: 10.2214/AJR.09.3193. PMid: 19843732.

[6] Coche E, Lonneux M, Geets X. Lung cancer: morphological and functional approach to screening, staging and treatment planning. Future Oncol 2010; 6: 367-380. doi: 10.2217/fon.10.7. PMid: 20222794.

[7] Murakami S, Saito H, Sakuma Y, et al. Correlation of ${ }^{18}$ F-fluorodeoxyglucose uptake on positron emission tomography with ki67 index and pathological invasive area in lung adenocarcinomas $30 \mathrm{~mm}$ or less in size. Eur J Radiol 2010; 75: e62-e66. doi: 10.1016/j.ejrad.2009.11.020. PMid: 20005653.

[8] Chang CY, Chang SJ, Chang SC, Yuan MK. The value of positron emission tomography in early detection of lung cancer in high risk population: a systematic review. Clin Respir J 2013; 7: 1-6. doi: 10.1111/j.1752-699X.2012.00290.x. PMid: 22404955.

[9] Cronin P, Dwamena BA, Kelly AM, Carlos RC. Solitary pulmonary nodules: meta-analytic comparison of cross-sectional imaging modalities for diagnosis of malignancy. Radiology 2008; 246: 772-782. doi: 10.1148/radiol.2463062148. PMid: 18235105.

[10] Schöder H, Gönen M. Screening for cancer with PET and PET/ CT: potential and limitations. J Nucl Med 2007; 48(Suppl 1): 4S-18S. doi: 10.2967/jnumed.107.044446. PMid: 17204716.

[11] Black C, de Verteuil R, Walker S, et al. Population screening for lung cancer using computed tomography, is there evidence of clinical effectiveness? A systematic review of the literature. Thorax 2007; 62: 131-138. doi: 10.1136/thx.2006.064659. PMid: 17287305. 
[12] Terauchi T, Murano T, Daisaki H, et al. Evaluation of wholebody cancer screening using ${ }^{18} \mathrm{~F}$-2-deoxy-2-fluoro-D-glucose positron emission tomography: a preliminary report. Ann Nucl Med 2008; 22: 379-385. doi: 10.1007/s12149-008-0130-7. PMid: 18600415

[13] Lee JW, Kang KW, Paeng JC, et al. Cancer screening using ${ }^{18} \mathrm{~F}$ FDG PET/CT in Korean asymptomatic volunteers: a preliminary report. Ann Nucl Med 2009; 23: 685-691. doi: 10.1007/ s12149-009-0291-z. PMid: 19662344.

[14] Whiting PF, Rutjes AW, Westwood ME, et al. QUADAS-2: a revised tool for the quality assessment of diagnostic accuracy studies. Ann Intern Med 2011; 155: 529-536. doi: 10.1059/ 0003-4819-155-8-201110180-00009. PMid: 22007046.

[15] Zamora J, Abraira V, Muriel A, Khan KS, Coomarasamy A. Meta-DiSc: a software for meta-analysis of test accuracy data. BMC Med Res Methodol 2006; 6: 31. doi: 10.1186/1471-22886-31. PMid: 16836745.

[16] Harbord RM, Whiting P. Metandi: meta-analysis of diagnostic accuracy using hierarchical logistic regression. Stata J 2009; 9: 211-229.

[17] Chen YK, Ding HJ, Su CT, et al. Application of PET and PET/ CT imaging for cancer screening. Anticancer Res 2004; 24 : 4103-4108. PMid: 15736459.

[18] Ide M. Cancer screening with FDG-PET. Q J Nucl Med Mol Imaging 2006; 50: 23-27. PMid: 16557201.

[19] Kao CH, Kwan AS, Kwan JK, Chow MJ. The role of ${ }^{18}$ F-fluorodeoxyglucose positron emission tomography in cancer screening-a preliminary report. Oncol Rep 2001; 8: 1145-1148. PMid: 11496332.

[20] Shibata K, Arai M, Matsuura M, et al. Relationship of detection rate of PET cancer screening examinees and risk factors: analysis of background of examinees. Ann Nucl Med 2011; 25: 261-267. doi: 10.1007/s12149-010-0458-7. PMid: 21188657.

[21] Nishizawa S, Kojima S, Teramukai S, et al. Prospective evaluation of whole-body cancer screening with multiple modalities including $\left[{ }^{18} \mathrm{~F}\right]$ fluorodeoxyglucose positron emission tomography in a healthy population: a preliminary report. J Clin Oncol 2009; 27: 1767-1773. doi: 10.1200/JCO.2008.18.2238. PMid: 19255324.

[22] Ono K, Ochiai R, Yoshida T, et al. The detection rates and tumor clinical/pathological stages of whole-body FDG-PET cancer screening. Ann Nucl Med 2007; 21: 65-72. doi: 10.1007/BF3034001. PMid: 17373338.

[23] Bastarrika G, Garcia-Velloso MJ, Lozano MD, et al. Early lung cancer detection using spiral computed tomography and positron emission tomography. Am J Respir Crit Care Med 2005; 171: 1378-1383. doi: 10.1164/rccm.200411-14790C.PMid: 15790860 .

[24] Pastorino U, Bellomi M, Landoni C, et al. Early lung-cancer detection with spiral $\mathrm{CT}$ and positron emission tomography in heavy smokers: 2-year results. Lancet 2003; 362: 593-597. doi: 10.1016/S0140-6736(03)14188-8. PMid: 12944057.

[25] Veronesi G, Bellomi M, Mulshine JL, et al. Lung cancer screening with low-dose computed tomography: a non-invasive diagnostic protocol for baseline lung nodules. Lung Cancer 2008; 61: 340-349. doi: 10.1016/j.lungcan.2008.01.001. PMid: 18308420.

[26] Ashraf H, Dirksen A, Loft A, et al. Combined use of positron emission tomography and volume doubling time in lung cancer screening with low-dose CT scanning. Thorax 2011; 66: 315-319. doi: 10.1136/thx.2010.136747. PMid: 21169285.

[27] van't Westeinde SC, de Koning HJ, Thunnissen FB, et al. The role of the ${ }^{18} \mathrm{~F}$-fluorodeoxyglucose-positron emission tomography scan in the Nederlands Leuvens Longkanker screenings Onderzoek lung cancer screening trial. J Thorac Oncol 2011; 6: 1704-1712. doi: 10.1097/JTO.0b013e3182286d0b. PMid: 21876458.

[28] Field JK, Smith RA, Aberle DR, et al. International Association for the Study of Lung Cancer Computed Tomography Screening Workshop 2011 report. J Thorac Oncol 2012; 7: 10-19. doi: 10.1097/JTO.0b013e31823c58ab. PMid: 22173661.

[29] Smith RA, Cokkinides V, Brooks D, Saslow D, Shah M, Brawley OW. Cancer screening in the United States, 2011: a review of current American Cancer Society Guidelines and issues in cancer screening. CA Cancer J Clin 2011; 61: 8-30. doi: 10.3322/caac.20096. PMid: 21205832.

[30] McMahon PM, Kong CY, Bouzan C, et al. Cost-effectiveness of computed tomography screening for lung cancer in the United States. J Thorac Oncol 2011; 6: 1841-1848. doi: 10.1097/ JTO.0b013e31822e59b3. PMid: 21892105.

[31] Bach PB, Jett JR, Pastorino U, Tockman MS, Swensen SJ, Begg CB. Computed tomography screening and lung cancer outcomes. JAMA 2007; 297: 953-961. doi: 10.1001/ jama.297.9.953. PMid: 17341709.

[32] Infante M, Chiesa G, Solomon D, et al. Surgical procedures in the DANTE trial, a randomized study of lung cancer early detection with spiral computed tomography: comparative analysis in the screening and control arm. J Thorac Oncol 2011; 6: 327-335. doi: 10.1097/JTO.0b013e318200f523. PMid: 21178639.

[33] Byrne MM, Koru-Sengul T, Zhao W, Weissfeld JL, Roberts MS. Healthcare use after screening for lung cancer. Cancer 2010; 116: 4793-4799. doi: 10.1002/cncr.25466. PMid: 20597136.

[34] Ma BB, Hui EP, Mok TS. Population-based differences in treatment outcome following anticancer drug therapies. Lancet Oncol 2010; 11: 75-84. doi: 10.1016/S1470-2045(09)70160-3. PMid: 20129130.

[35] Deppen S, Putnam Jr JB, Andrade G, et al. Accuracy of FDGPET to diagnose lung cancer in a region of endemic granulomatous disease. Ann Thorac Surg 2011; 92: 428-433. doi: 10.1016/j.athoracsur.2011.02.052. PMid: 21592456. 